\title{
The influence of spectral solar irradiance data on stratospheric heating rates during the 11 year solar cycle
}

\author{
S. Oberländer, ${ }^{1}$ U. Langematz, ${ }^{1}$ K. Matthes, ${ }^{1,2}$ M. Kunze, ${ }^{1}$ A. Kubin,,${ }^{1}$ J. Harder, ${ }^{3}$ \\ N. A. Krivova, ${ }^{4}$ S. K. Solanki, ${ }^{4,5}$ J. Pagaran, ${ }^{6}$ and M. Weber ${ }^{6}$ \\ Received 9 September 2011; revised 22 November 2011; accepted 24 November 2011; published 4 January 2012.
}

[1] Heating rate calculations with the FUBRad shortwave (SW) radiation parameterization have been performed to examine the effect of prescribed spectral solar fluxes from the NRLSSI, MPS and IUP data sets on SW heating rates over the 11 year solar cycle 22 . The corresponding temperature response is derived from perpetual January General Circulation Model (GCM) simulations with prescribed ozone concentrations. The different solar flux input data sets induce clear differences in SW heating rates at solar minimum, with the established NRLSSI data set showing the smallest solar heating rates. The stronger SW heating in the middle and upper stratosphere in the MPS data warms the summer upper stratosphere by $2 \mathrm{~K}$. Over the solar cycle, SW heating rate differences vary up to $40 \%$ between the irradiance data sets, but do not result in a significant change of the solar temperature signal. Lower solar fluxes in the newer SIM data lead to a significantly cooler stratosphere and mesosphere when compared to NRLSSI data for 2007. Changes in SW heating from 2004 to 2007 are however up to six times stronger than for the NRLSSI data. Citation: Oberländer, S., U. Langematz, K. Matthes, M. Kunze, A. Kubin, J. Harder, N. A. Krivova, S. K. Solanki, J. Pagaran, and M. Weber (2012), The influence of spectral solar irradiance data on stratospheric heating rates during the 11 year solar cycle, Geophys. Res. Lett., 39, L01801, doi:10.1029/2011GL049539.

\section{Introduction}

[2] Earth's atmosphere responds to solar irradiance changes, both directly by the radiative forcing and indirectly through dynamical feedbacks (see Gray et al. [2010] for a review). While total solar irradiance (TSI) between the minimum (min) and maximum (max) phases of the 11 year solar cycle varies only by about $0.1 \%$, variations of several to several tens percent have been measured in the ultraviolet (UV) spectral region [e.g., Lean, 2000]. According to Krivova et al. [2006], wavelengths between $200 \mathrm{~nm}$ and $400 \mathrm{~nm}$ account for $60 \%$ of the 11 year solar cycle variations. Higher UV input during solar max enhances photochemical ozone production in the stratosphere [e.g., Haigh,

\footnotetext{
${ }^{1}$ Institut für Meteorologie, Freie Universität Berlin, Berlin, Germany.

${ }^{2}$ Helmholtz-Zentrum Potsdam Deutsches GeoForschungsZentrum, Potsdam, Germany.

${ }^{3}$ Laboratory for Atmospheric and Space Physics, University of Colorado at Boulder, Boulder, Colorado, USA

${ }^{4}$ Max-Planck-Institut für Sonnensystemforschung, Katlenburg-Lindau, Germany.

${ }^{5}$ School of Space Research, Kyung Hee University, Yongin, South Korea.

${ }^{6}$ Institut für Umweltphysik, Universität Bremen, Bremen, Germany.
}

Copyright 2012 by the American Geophysical Union. 0094-8276/12/2011GL049539
1994] and causes stronger heating through absorption of solar photons by molecular oxygen and ozone [e.g., Matthes et al., 2004].

[3] Therefore, the simulation of solar effects on the atmosphere in global GCMs or Chemistry-Climate Models (CCMs) requires shortwave (SW) radiation schemes that account for spectral variations of the solar irradiance [Nissen et al., 2007; Forster et al., 2011]. The amplitude of the simulated solar signal depends on the spectral solar fluxes prescribed at the top of the atmosphere (TOA). Differences in the solar irradiance spectrum incident at the TOA were shown to affect SW heating rates in the middle and upper stratosphere. Using spectral data from a theoretical spectral line model and the NRLSSI data in a line-by-line model Zhong et al. [2008] found differences of up to $1.1 \mathrm{~K} /$ day in mid-latitude summer.

[4] Several spectral data sets for different solar cycles from various measurement platforms are now available. Most widely used is the Naval Research Laboratory Spectral Solar Irradiance (NRLSSI) data set that is based on an empirical model adjusted to measurements from TIMED/ SEE (Thermosphere Ionosphere Mesosphere Energetics and Dynamics - Solar EUV Experiment), SOLSTICE (Solar Stellar Irradiance Comparison Experiment) on board UARS (Upper Atmosphere Research Satellite), and SOLSPEC (Solar Spectral Irradiance Measurements) [Lean, 2000; Lean et al., 2005]. An alternative, daily total and spectral solar flux time series has been reconstructed from SOHO (Solar and Heliospheric Observatory) MDI (Michelson Doppler Imager) and KPNSO (Kitt Peak National Solar Observatory) full-disk magnetograms, using the SATIRE (Spectral and Total Irradiance Reconstructions) model [Krivova et al., 2009, 2011]. Daily total and spectral solar fluxes have also been measured by SCIAMACHY (Scanning Imaging Absorption Spectrometer for Atmospheric Chartography) on board the Environmental Satellite (ENVISAT) and were used together with solar proxies to infer solar cycle changes [Pagaran et al., 2009]. More recently, Harder et al. [2009] presented measurements from the Spectral Irradiance Monitor (SIM) aboard SORCE that suggest up to six times larger changes in UV in the years 2004 to 2007 than the NRLSSI data. In addition, the visible (VIS) and near-infrared (NIR) variations in the SIM data were out of phase to the changes in TSI and UV, with increasing irradiance towards the minimum of solar cycle number 23. Such a spectral shift in the decadal solar signal with more UV but less VIS and NIR energy at solar max might change the sign of the radiative forcing of the troposphere-surface system [Haigh et al., 2010] and also affect the stratospheric solar temperature signal [Cahalan et al., 2010].

[5] The purpose of this study is to examine, to which extent the solar signal in a model is affected by the prescribed TOA solar fluxes. Using a SW radiation scheme with enhanced 
Table 1. Spectral Solar Flux Input Data Sets

\begin{tabular}{lcccc}
\hline Name & Source & Spectral Range & Sampling & Reference \\
\hline NRLSSI & TIMED/SEE, SOLSTICE, SOLSPEC with empirical model & $0-3000 \mathrm{~nm}$ & $1-5 \mathrm{~nm}$ & Lean [2000], Lean et al. [2005] \\
MPS & KPNSO and MDI with SATIRE model & $115-160000 \mathrm{~nm}$ & $1 \mathrm{~nm}$ & Krivova et al. [2009, 2011] \\
IUP & SCIAMACHY with SCIA proxy model & $230-1750 \mathrm{~nm}$ & $1 \mathrm{~nm}$ & Pagaran et al. [2009] \\
SIM & SIM (SOLSTICE $<200 \mathrm{~nm})$ & $200-2412 \mathrm{~nm}$ & $1-34 \mathrm{~nm}$ & Harder et al. [2009] \\
\hline
\end{tabular}

spectral resolution the impact of different spectral input data sets on the stratospheric heating for an atmospheric basic state (solar min conditions), and the 11 year solar cycle is compared. The radiative-dynamical response of the atmosphere to solar flux variations is studied here, while chemical changes in stratospheric ozone by solar flux variations and their feedback on the radiative budget are not considered.

[6] In Section 2 the experimental setup and the irradiance data sets are introduced. In Section 3 the results of the SW heating rate calculations for solar min conditions and 11 year solar cycle variations, as well as the temperature response from a full GCM simulation are presented. Section 4 includes a summary and a discussion.

\section{Experiments and Irradiance Data Sets}

[7] Offline SW heating rate calculations were performed with the FUBRad SW radiation parameterization using different spectral irradiance data sets as input. FUBRad [Nissen et al., 2007] resolves the UV and VIS spectral range with 49 intervals between 121.56 and $683 \mathrm{~nm}$ in the height range between $70 \mathrm{hPa}(\sim 20 \mathrm{~km})$ and $0.01 \mathrm{hPa}(\sim 80 \mathrm{~km})$. The NIR is represented in three spectral bands between 690 and $4000 \mathrm{~nm}$ [Roeckner et al., 2003]. At pressures higher than $70 \mathrm{hPa}$ the scheme is coupled to the parameterization by Fouquart and Bonnel [1980]. FUBRad allows detailed studies on the influence of solar variability and has a high skill compared to a line-by-line reference model [Forster et al., 2011]. For the solar cycle number 22, SW heating rate differences between solar min (September 1986) and solar max (November 1989) were calculated, following the recommendations of Forster et al. [2011]. Orbital parameters and the solar zenith angles were fixed for January 15. A zonal mean ozone climatology for January [Fortuin and Langematz, 1994] was prescribed.

[8] We compared in a first step three spectral irradiance data sets: the SOLSTICE/SOLSPEC based NRLSSI data as a reference, the magnetogram based data of the Max-PlanckInstitut für Sonnensystemforschung (hereafter called MPS data), and the SCIAMACHY measurements of the Institut für Umweltphysik (IUP), Bremen (hereafter called IUP data). Table 1 (first three rows) summarizes the details of the data sets with respective references. IUP data were extended by MPS data below $230 \mathrm{~nm}$. The high resolution data were integrated over the FUBRad spectral intervals. A comparison of the incoming fluxes at solar min (September 1986) for the three input data sets in the Hartley and Huggins spectral intervals of FUBRad (cf. Figure S1 in the auxiliary material) shows that the IUP and NRLSSI data sets show pretty good agreement, with slightly higher IUP values in some of the Hartley bands (206.5-277.8 nm) and some of the Huggins bands $(277.8-362.5 \mathrm{~nm}){ }^{1}$ The MPS data show larger deviations from the other two data sets particularly in the

\footnotetext{
${ }^{1}$ Auxiliary materials are available in the HTML. doi:10.1029/ 2011 GL049539.
}

Huggins bands with higher solar fluxes in those SW parts of the bands that are most relevant for ozone absorption.

[9] Corresponding temperature changes were derived from simulations with the ECHAM/MESSy Atmospheric Chemistry (EMAC) model [Jöckel et al., 2006]. In the EMAC-FUB configuration used here, the model includes the FUBRad radiation scheme and consists of 39 layers up to $0.01 \mathrm{hPa}(\sim 80 \mathrm{~km})$. To identify the solar signal, 50 months under perpetual January conditions were calculated for solar min and solar max irradiances respectively, using the NRLSSI and MPS solar flux data as input. A comparison with a previous 20-year GCM run including the full annual cycle had shown that the thermal response in the summer hemisphere to the prescribed irradiance changes was not affected by perpetual season conditions (not shown). Moreover, integrating over 50 Januaries improved the ensemble for the statistical analyses of the different simulations. As our study focused on the pure radiative-dynamical effects of irradiance variations, the model was run in GCM mode, i.e. without interactive chemistry, using a prescribed fixed ozone climatology [Fortuin and Langematz, 1994]. Hence the solar response is forced by irradiance changes only, without considering an ozone feedback, the consequences of which will be discussed later.

[10] In a second step, we studied the implications of UV and VIS irradiance changes as suggested by the Spectral Irradiance Monitor (SIM) on board the Solar Radiation and Climate Experiment (SORCE) satellite [Harder et al., 2009] (hereinafter referred to as SIM data) on solar heating rates and temperature. Below $200 \mathrm{~nm}$ SOLSTICE data were added. We used available data from May 2004 to November 2007 roughly corresponding to the transition from the mean to the minimum of solar cycle 23 . Table 1 provides further information on the SIM data.

\section{Results}

\subsection{Solar Minimum Conditions}

[11] SW heating rates for solar min conditions calculated with FUBRad using the NRLSSI data show maximum heating of about $14 \mathrm{~K} /$ day around the summer stratopause at about $50 \mathrm{~km}$ altitude (Figure 1a). A secondary heating maximum occurs in the upper mesosphere due to absorption in the Schumann-Runge bands. The results are in agreement with earlier studies [e.g., Nissen et al., 2007]. Using the MPS and IUP data yields mostly higher heating rates compared to the NRLSSI data. In the middle to upper stratosphere between 30 and $50 \mathrm{~km}$ the largest SW heating rates of the three data sets are produced by the MPS data set. Differences between the MPS and NRLSSI data reach $0.2 \mathrm{~K} /$ day or nearly $5 \%$ in the global mean (Figures $1 \mathrm{~b}$ and 1c), with a maximum of $0.35 \mathrm{~K} /$ day or about $6 \%$ in the summer stratosphere at $5 \mathrm{hPa}$ (not shown). Heating rates derived from the IUP data are slightly higher in the upper stratosphere and lower mesosphere (around $0.1 \mathrm{~K} /$ day or $1 \%$ ) but lower in the 

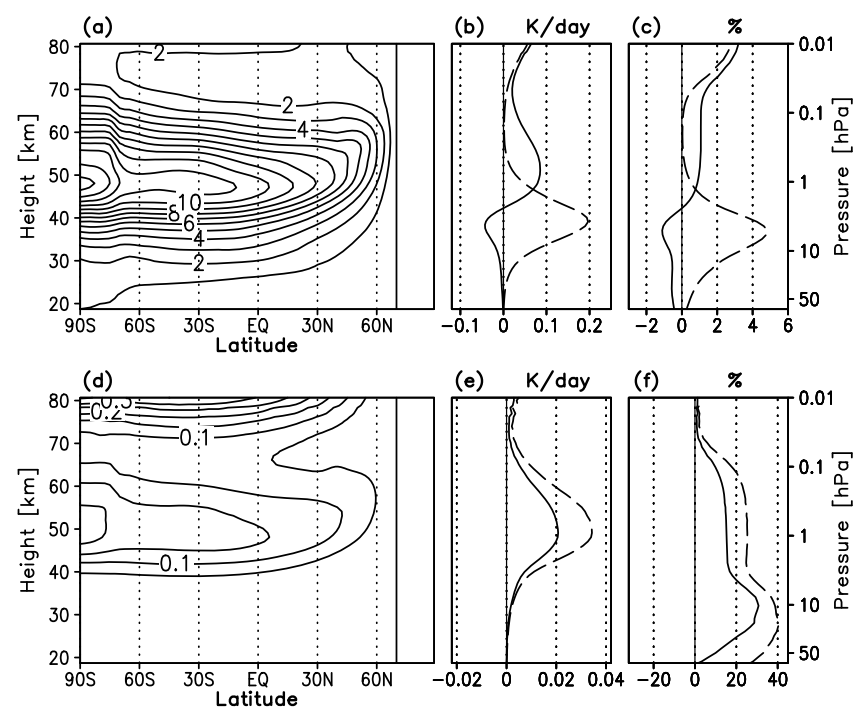

Figure 1. (top) Solar heating rates in K/d for the NRLSSI data at solar min and (bottom) solar cycle differences: (a and d) zonal mean NRLSSI data, (b and e) global mean differences in K/d for IUP-NRLSSI (solid) and MPS-NRLSSI (dashed), and (c and f) same as Figures $1 \mathrm{~b}$ and $1 \mathrm{e}$ but in $\%$ of the NRLSSI data.

lower and middle stratosphere. Above $0.05 \mathrm{hPa}$ solar heating rates derived from the IUP and the MPS data sets are similar, as for wavelengths shorter than $230 \mathrm{~nm}$ the same input data were used. For solar max conditions the differences are nearly the same but with slightly higher absolute values (not shown). The variation in heating rates between the three data sets can be explained by the differences in the derived incoming spectral fluxes (see Figure S1). The stronger SW heating in the MPS data below the heating maximum is caused by an enhanced solar flux in the MPS data in the
Huggins bands that mostly heat the middle stratosphere, while the stronger heating rates in the IUP data are due to stronger solar fluxes in the Hartley bands leading to increased heating around the stratopause.

[12] To infer the temperature response to the heating rate differences from the spectral data sets, simulations with the EMAC-FUB model have been performed using the two data sets with the largest discrepancies in SW heating rates, NRLSSI and MPS. Most of the stratosphere is significantly warmer at solar min when MPS data are used (Figure 2a). The higher temperatures extend from the summer pole with a maximum of about $2 \mathrm{~K}$ to the subtropics of the winter hemisphere. They also spread into the high latitude upper summer mesosphere. As the thermal structure in these regions is determined by radiative processes, these temperature changes directly reflect the impact of the enhanced solar fluxes in the MPS data set. The temperature changes in the polar winter stratosphere are not significant and presumably influenced by internal dynamical variability.

\subsection{Year Solar Cycle Variations}

[13] Figure 1d shows differences in solar heating rates between the min and max phases of the 11 year solar cycle for the NRLSSI data. The largest stratospheric solar signal of about $0.2 \mathrm{~K} /$ day occurs around $50 \mathrm{~km}$ at the summer stratopause. The strong increase in heating rates of up to $0.3 \mathrm{~K} /$ day in the upper mesosphere is caused by stronger absorption of the Lyman- $\alpha$ line by molecular oxygen at solar max.

[14] Heating rates calculated using MPS data show the highest increase from solar min to max of all data sets. The global mean solar cycle difference for the MPS data is about $0.03 \mathrm{~K} /$ day stronger than for the NRLSSI data (Figure 1e), exceeding the maximum NRLSSI heating rate difference at the summer pole around $50 \mathrm{~km}$ by $0.05 \mathrm{~K} /$ day (not shown). 11 year solar cycle variations in SW heating rates from IUP data exceed those from the NRLSSI data as well, however, less than the MPS data. Although these absolute solar
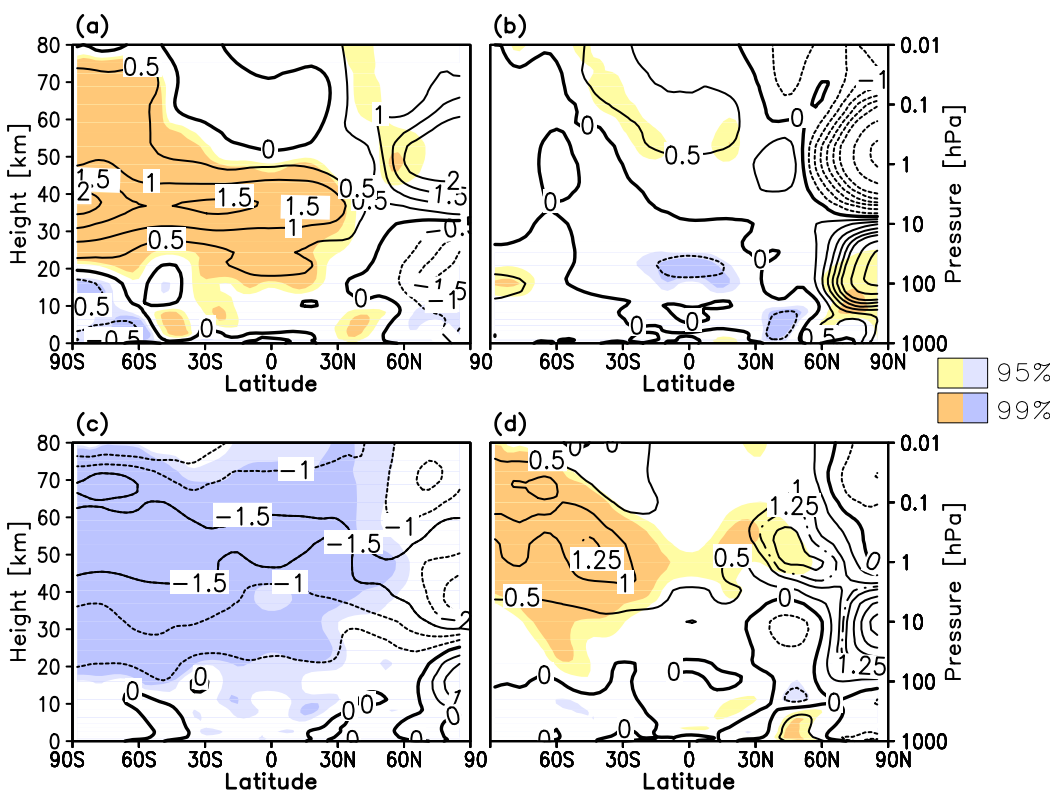

Figure 2. Zonal mean temperature difference in K between the MPS and NRLSSI data (a) at solar min and (b) for the 11 year solar cycle difference and between the SIM and NRLSSI data (c) in 2007 and (d) 2004 minus 2007. Light (dark) shading shows statistical significance at 95\% (99\%). 


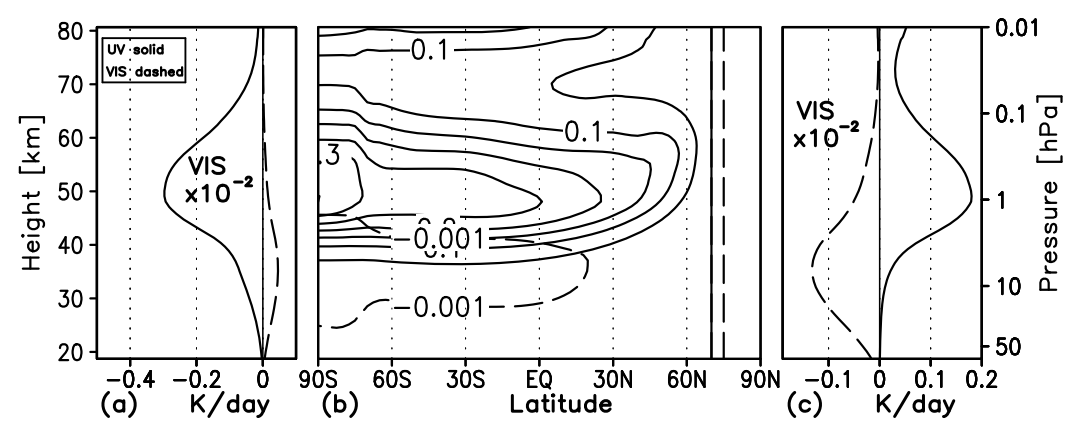

Figure 3. Solar heating rates: (a) global mean difference between SIM and NRLSSI in 2007, (b) zonal mean difference in 2004-2007 from SIM data, and (c) global mean difference in 2004-2007 change between SIM and NRLSSI data.

heating rate differences between the data sets seem to be small, both the IUP and MPS data produce a solar cycle heating signal that is between 20 and $40 \%$ larger than the NRLSSI data in the middle and lower stratosphere and between 10 and $20 \%$ larger in the upper stratosphere and mesosphere (Figure 1f). Changing the solar flux input data from NRLSSI to MPS has no significant effect on the solar temperature signal in the stratosphere (Figure 2b). Only two small statistically significant areas emerge which are not related directly to the solar heating rate changes. The $0.5 \mathrm{~K}$ lower temperature derived from MPS data in the tropical middle stratosphere is rather induced by dynamical warming in the winter polar stratosphere that in the GCM simulation is strongly forced by internal dynamical variability.

\subsection{Solar Signal From SIM Irradiances}

[15] UV changes in the SIM data from 2004 to 2007, i.e. about half the 11 year solar cycle, exceed those in other data sets by a factor four to six [e.g., Harder et al., 2009; Pagaran et al., 2011]. The differences in the decline of UV radiation between the SIM and other data are much larger than the differences in the UV change between the NRLSSI and the MPS or IUP data for the complete solar cycle. Furthermore, solar irradiance from SIM increases in the VIS and NIR spectral range towards solar min, opposite to the UV variation. Here we compare SW heating rates for the SIM and NRLSSI data calculated with FUBRad. Figure 3a presents heating rate differences between the two data sets for the solar min in November 2007, separated for the UV and VIS spectral ranges. NIR changes could not be assessed as the EMAC NIR scheme does not use spectral input. The latitudeheight structure of the SIM solar heating rates is very similar to that from NRLSSI data for solar min in 1986 (Figure 1a), however, due to lower absolute irradiances heating in the UV bands is weaker for the SIM data throughout the middle atmosphere (Figure 3a). The maximum heating deficit in the SIM data of $0.5 \mathrm{~K} /$ day at the summer polar stratopause is of comparable magnitude to the discrepancy between MPS and NRLSSI but of opposite sign. In the Chappuis band SIM data produce slightly higher heating rates for solar min. SIM irradiances lead to a statistically significant cooler stratosphere and mesosphere in summer by up to $1.5 \mathrm{~K}$ (Figure 2c).

[16] The change in solar heating rate between 2004 and 2007 from SIM data is characterized by a decrease in UV irradiance, associated with a reduction of solar heating by up to $0.3 \mathrm{~K} /$ day at the summer stratopause towards low solar activity in 2007 (Figure 3b). In the VIS range the SIM data produce an increase in heating towards lower solar activity.
However, due to the weak absorption in the Chappuis band, changes in the VIS range over the solar cycle are two orders of magnitude smaller and do not reverse the UV induced decrease in radiative heating towards 2007.

[17] Compared to NRLSSI, the 2004-2007 solar signal is by a factor of six stronger in the SIM data. The SIM solar signal exceeds that from NRLSSI by $0.28 \mathrm{~K} /$ day at the summer stratopause (not shown) and $0.18 \mathrm{~K} /$ day in the global mean (Figure 3c). Corresponding changes are found in temperature with a statistically significant warmer summer upper stratosphere and mesosphere in 2004 than in 2007 when using the SIM data (Figure 2d). The difference in the solar temperature signal between SIM and NRLSSI is positive over nearly the whole depth of the stratosphere (Figure 2d) as the negative contribution from the VIS flux is more than compensated by the UV increase.

\section{Summary and Discussion}

[18] The comparison of three different solar flux input data sets (NRLSSI, MPS and IUP) used in the FUBRad SW radiation scheme has revealed clear differences in SW heating rates for the cycle 22 solar minimum (September 1986). The NRLSSI data show the smallest solar heating rates. The MPS data lead to up to $5 \%$ stronger solar heating in the middle and upper stratosphere, while the IUP data slightly enhance solar heating in the mesosphere. The differences come from stronger solar fluxes in the Huggins bands of the MPS data, and enhanced fluxes in the Hartley bands of the IUP data. Exchanging the NRLSSI with the MPS solar flux data in a GCM simulation may lead to a warmer summer stratosphere and mesosphere, reaching $2 \mathrm{~K}$ at the summer stratopause in the EMAC-FUB model of our study.

[19] SW heating rate differences between solar min and max for the three data sets vary by up to $40 \%$ in the lower and middle stratosphere and around $20 \%$ in the upper stratosphere and mesosphere. The absolute irradiances for past solar cycles have been derived or 'reconstructed' using models to calibrate measurements taken later with different instruments, like for example SOLSTICE, Atlas 3, or SUSIM. Harder et al. [2010] and Pagaran et al. [2011] showed that the instruments agree to about $2-4 \%$ depending on wavelength, which does not seem to be sufficient to detect potential future irradiance trends over multiple solar cycles. It is therefore important to narrow down the uncertainties in the measurements. As for the given solar flux data, no significant effect on the solar temperature signal could be found. 
[20] Larger changes in solar heating rates and resulting temperatures are found when using SIM measurements as input data. At solar min, SIM data heat the summer stratosphere and mesosphere by a maximum of $1.5 \mathrm{~K}$ less than the NRLSSI data. This is due to lower UV in the SIM data than empirically derived in the NRLSSI data. However, when considering the solar heating rate change between 2004 (medium solar activity) and 2007 (solar min), the solar signal from the SIM data is stronger by up to $0.18 \mathrm{~K} /$ day in the global mean due to larger UV flux differences compared to the NRLSSI data. The lower VIS irradiance (and the associated relative radiative cooling) at higher solar activity in SIM does not reverse the increase in total radiative heating. Overall, the SIM data cause a six times larger heating rate difference between the mean and minimum phases of the solar cycle 23 than the NRLSSI data. A projection on the full solar cycle 23 (scaled by the Mg II index), would correspond to a 2.4-fold higher solar heating signal in the SIM data, and consequently lead to a higher solar cycle temperature response of maximum $3 \mathrm{~K}$ in the upper stratosphere compared to NRLSSI and previous model simulations [e.g., Gray et al., 2010].

[21] Our estimate of the temperature response to the solar data sets is based on the direct effects of the irradiance variations on the radiative budget only hence neglecting the ozone-temperature feedback. Previous simulations with our GCM have shown that prescribing a stratospheric solar ozone signal of maximum $4 \%$ as derived from observations [e.g., Gray et al., 2010] results in a temperature signal around $0.5 \mathrm{~K}$ in the polar summer stratosphere with no significant signal at middle and low latitudes (not shown). As the differences in the solar cycle induced ozone signal are much smaller between the MPS, IUP and NRLSSI data sets respectively than between solar min and max, a temperature error less than $0.5 \mathrm{~K}$ is expected. As for the comparison SIM vs. NRLSSI, the significant temperature signal of around $1.25 \mathrm{~K}$ in the upper stratosphere of our GCM simulations agrees well with Haigh et al. [2010], while it is lower in the middle stratosphere. This is consistent with the calculated ozone response to the SIM irradiance variations of Haigh et al. [2010] showing the well-known ozone increase with rising irradiance in the middle stratosphere but a decrease above.

[22] Acknowledgments. We thank J. Lean for providing the NRLSSI data and P. Jöckel for support with EMAC. The work was funded by the DFG projects LA $1025 / 51-3$, SO $711 / 1-3$ and SOLOZON, and by the Korean grant R31-10016. The Mg II index was obtained from http:// www.swpc.noaa.gov/ftpmenu.

[23] The Editor thanks two anonymous reviewers for their assistance in evaluating this paper.

\section{References}

Cahalan, R. F., G. Wen, J. W. Harder, and P. Pilewskie (2010), Temperature response to spectral solar variability on decadal time scales, Geophys. Res. Lett., 37, L07705, doi:10.1029/2009GL041898.

Forster, P. M., et al. (2011), Evaluation of radiation scheme performance within chemistry climate models, J. Geophys. Res., 116, D10302, doi:10.1029/2010JD015361.

Fortuin, J. P. F., and U. Langematz (1994), An update on the global ozone climatology and on concurrent ozone and temperature trends, SPIE Atmos. Sens. Model., 2311, 207-216.
Fouquart, Y., and B. Bonnel (1980), Computations of solar heating of the Earth's atmosphere: A new parameterization, Contrib. Atmos. Phys., 53(1), 35-62.

Gray, L. J., et al. (2010), Solar influences on climate, Rev. Geophys., 48, RG4001, doi:10.1029/2009RG000282.

Haigh, J. D. (1994), The role of stratospheric ozone in modulating the solar radiative forcing of climate, Nature, 370, 544-546, doi:10.1038/370544a0.

Haigh, J. D., A. R. Winning, R. Toumi, and J. W. Harder (2010), An influence of solar spectral variations on radiative forcing of climate, Nature, 467, 696-699, doi:10.1038/nature09426.

Harder, J. W., J. M. Fontenla, P. Pilewskie, E. C. Richard, and T. N. Woods (2009), Trends in solar spectral irradiance variability in the visible and infrared, Geophys. Res. Lett., 36, L07801, doi:10.1029/2008GL036797.

Harder, J. W., et al. (2010), The SORCE SIM solar spectrum. Comparison with recent observations, Sol. Phys., 263, 3-24, doi:10.1007/s11207010-9555-y.

Jöckel, P., H. Tost, A. Pozzer, C. Brühl, J. Buchholz, L. Ganzeveld, P. Hoor, A. Kerkweg, and M. Lawrence (2006), The atmospheric chemistry general circulation model ECHAM5/MESSy: Consistent simulation of ozone from the surface to the mesosphere, Atmos. Chem. Phys., 6, 5067-5104, doi:10.5194/acp-6-5067-2006.

Krivova, N. A., S. K. Solanki, and L. Floyd (2006), Reconstruction of solar UV irradiance in cycle 23, Astron. Astrophys., 452, 631-639, doi:10.1051/0004-6361:20064809.

Krivova, N. A., S. K. Solanki, T. Wenzler, and B. Podlipnik (2009), Reconstruction of solar UV irradiance since 1974, J. Geophys. Res., 114, D00I04, doi:10.1029/2009JD012375.

Krivova, N. A., S. K. Solanki, and Y. Unruh (2011), Towards a long-term record of solar total and spectral irradiance, J. Atmos. Sol. Terr. Phys., 73, 223-234, doi:10.1016/j.jastp.2009.11.013.

Lean, J. L. (2000), Evolution of the Sun's spectral irradiance since the Maunder Minimum, Geophys. Res. Lett., 27(16), 2425-2428, doi:10.1029/ 2000 GL000043.

Lean, J. L., G. J. Rottmann, J. Harder, and G. Kopp (2005), SORCE contributions to new understanding of global change and solar variability, Sol. Phys., 230, 27-53, doi:10.1007/s11207-005-1527-2.

Matthes, K., U. Langematz, L. J. Gray, K. Kodera, and K. Labitzke (2004), Improved 11-year solar signal in the Freie Universität Berlin Climate Middle Atmosphere Model (FUB-CMAM), J. Geophys. Res., 109, D06101, doi:10.1029/2003JD004012.

Nissen, K. M., K. Matthes, U. Langematz, and B. Mayer (2007), Towards a better representation of the solar cycle in general circulation models, Atmos. Chem. Phys., 7, 5391-5400, doi:10.5194/acp-7-5391-2007.

Pagaran, J., M. Weber, and J. Burrows (2009), Solar variability from 240 to $1750 \mathrm{~nm}$ in terms of faculae brightening and sunspot darkening from SCIAMACHY, Astrophys. J., 700(2), 1884-1895, doi:10.1088/0004$637 \mathrm{X} / 700 / 2 / 1884$.

Pagaran, J., M. Weber, M. DeLand, L. Floyd, and J. P. Burrows (2011), Solar spectral irradiance variations in $240-1600 \mathrm{~nm}$ during the recent solar cycles 21-23, Sol. Phys., 1, 159-188, doi:10.1007/s11207-0119808-4.

Roeckner, E., et al. (2003), The atmospheric general circulation model ECHAM 5. Part I: Model description, MPI-Rep. 349, 127 pp., Max Planck Inst. for Meteorol., Hamburg, Germany.

Zhong, W., S. M. Osprey, L. J. Gray, and J. D. Haigh (2008), Influence of the prescribed solar spectrum on calculations of atmospheric temperature, Geophys. Res. Lett., 35, L22813, doi:10.1029/2008GL035993.

J. Harder, Laboratory for Atmospheric and Space Physics, University of Colorado at Boulder, Campus Box 590, Boulder, CO 80303-0590, USA.

N. A. Krivova and S. K. Solanki, Max-Planck-Institut für Sonnensystemforschung, Max-Planck-Str. 2, D-37191 Katlenburg-Lindau, Germany.

A. Kubin, M. Kunze, U. Langematz, and S. Oberländer, Institut für Meteorologie, Freie Universität Berlin, Carl-Heinrich-Becker-Weg 6-10, D-12165 Berlin, Germany. (ulrike.langematz@met.fu-berlin.de)

K. Matthes, Helmholtz-Zentrum Potsdam Deutsches GeoForschungsZentrum, Telegrafenberg C223, D-14473 Potsdam, Germany.

J. Pagaran and M. Weber, Institut für Umweltphysik, Universität Bremen, Otto-Hahn-Allee 1, D-28334 Bremen, Germany. 